\title{
Can the transformation time in phase change optical recording be improved by using femtosecond laser pulses?
}

\author{
J. Solis, S. M. Wiggins and C. N. Afonso. Instituto de Óptica, CSIC, Serrano 121, 28006-Madrid, SPAIN.
}

\begin{abstract}
Non-stoichiometric, Sb-rich, amorphous thin films, such as $\mathrm{Sb}_{\mathrm{x}} \mathrm{Ge}_{1-\mathrm{x}}$ with $\mathrm{x}>0.85$ have been demonstrated to be promising candidates for the development of ultrashort laser pulse driven rewritable phase change optical disks [1]. Depending on the configuration, these materials can be reversibly crystallised and re-amorphised under pico- and femtosecond laser pulses while exhibiting optical contrast. Cyclability under ultrashort pulses has been proven possible through the control of the solidification dynamics after laser induced melting. At the high fluences required for reamorphisation, if there is a large difference between the thermal conductivity of the liquid phase of the film and that of the substrate material, the nucleation of the solid takes place throughout the whole molten volume with an enormous nucleation rate. As a consequence of this bulk solidification process, the massive release of solidification enthalpy and the subsequent decrease of supercooling (recalescence process) can frustrate re-amorphisation. The negative effects of recalescence can however be compensated for with appropriate selection of the film thickness. In such a way, full transformation times of the order of $400 \mathrm{ps}$ for both the crystallisation and amorphisation processes have been demonstrated in GeSb films with a thickness of $25 \mathrm{~nm}$ upon irradiation with $30 \mathrm{ps}$ laser pulses at $580 \mathrm{~nm}$ [2].
\end{abstract}

In this work we have extended our previous studies in order to investigate the joint influence of the film thickness, wavelength and pulse duration on the transformation time between the crystalline and the amorphous phases of GeSb films under picosecond and femtosecond laser pulses aiming to find routes for further improvements of the transformation time. We have used tunable laser pulses delivered by an optical parametric amplifier (OPA) pumped by a $\mathrm{Ti}: \mathrm{Al}_{2} \mathrm{O}_{3}$ regenerative amplifier to induce the re-amorphisation process. The duration of the pulses from the OPA has been varied from approximately $70 \mathrm{fs}$ up to $20 \mathrm{ps}$ by using conventional pulse stretching techniques. The samples used have been $\mathrm{Ge}_{0.07} \mathrm{Sb}_{0.93}$ films with a thickness ranging from 10 to $50 \mathrm{~nm}$ grown in a DC magnetron sputtering system on glass substrates at room temperature. The transformation time has been measured by analysing the time evolution of the reflectivity of the irradiated regions in a single shot experiment using a set-up based on the use of a streak camera operating in single sweep mode [1,2]. Depending on the time window chosen, the time resolution of the reflectivity measurements can range from approximately $350 \mathrm{ps}$ down to $1 \mathrm{ps}$.

The results obtained so far clearly indicate that, for a given film thickness, the use of pulses in the pico- or femto-second regimes at the same wavelength induces a strong modification in the solidification scenario. Films with a thickness of $25 \mathrm{~nm}$ irradiated with $20 \mathrm{ps}$ laser pulses at $600 \mathrm{~nm}$ show a transformation time for re-amorphisation below 400 ps while the same films irradiated with femtosecond laser pulses re-amorphise in a much longer time, of the order of $10 \mathrm{~ns}$. Upon shifting the wavelength to the near infrared region $(780-800 \mathrm{~nm})$, transformation times for both 70 fs and 20 ps pumping are of the order of $10 \mathrm{~ns}$. We interpret this behaviour as a clear indication that both parameters (pulse duration and wavelength) strongly affect, among other factors which will be discussed, the degree of supercooling prior to solidification, hence the large variations in the solidification mechanism and subsequent transformation time.

[1] J. Solis and C. N. Afonso, Ultrashort-laser-pulse-driven rewriteable phase-change optical recording in Sb-based films, Appl.Phys. A76, 331-338 (2003).

[2] J. Siegel, C. N. Afonso and J. Solis, Dynamics of ultrafast reversible phase transitions in GeSb films triggered by picosecond pulses, Appl.Phys.Lett. 75, 3102-3104 (1999). 